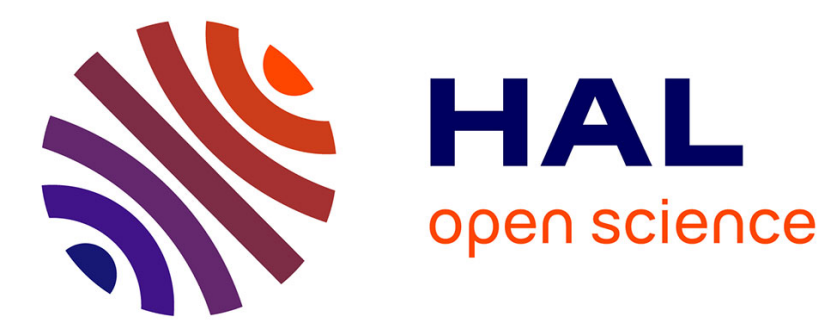

\title{
Templates of Two Foliated Attractors - Lorenz and Chen Systems
}

\author{
Martin Rosalie
}

\section{To cite this version:}

Martin Rosalie. Templates of Two Foliated Attractors - Lorenz and Chen Systems. International journal of bifurcation and chaos in applied sciences and engineering , 2016, 26 (02), pp.1650037. 10.1142/S0218127416500371. hal-01009552

\section{HAL Id: hal-01009552 https://hal.science/hal-01009552}

Submitted on 18 Jun 2014

HAL is a multi-disciplinary open access archive for the deposit and dissemination of scientific research documents, whether they are published or not. The documents may come from teaching and research institutions in France or abroad, or from public or private research centers.
L'archive ouverte pluridisciplinaire HAL, est destinée au dépôt et à la diffusion de documents scientifiques de niveau recherche, publiés ou non, émanant des établissements d'enseignement et de recherche français ou étrangers, des laboratoires publics ou privés. 


\title{
TEMPLATES OF TWO FOLIATED ATTRACTORS - LORENZ AND CHEN SYSTEMS
}

\author{
M. Rosalie*
}

\begin{abstract}
A chaotic attractor solution of the Lorenz system [1] with foliated structure is topologically characterized. Its template permits to both summarize the organization of its periodic orbits and detail the topology of the solution as a branched manifold. A template of an attractor solution of the Chen system [2] with a similar foliated structure is also established.
\end{abstract}

Keywords. Chaotic attractor, topological characterization, template

\section{Introduction}

The Lorenz system has been introduced with a set of parameters [1] for which the solution is a chaotic attractor. The topology of this solution has been established by Birman \& Williams [3] considering the periodic orbits of the solution as knots evolving in a branched manifold, currently called template. From experimental or numerical data, the idea of using periodic orbits to characterize chaotic attractors comes from Auerbach et al. [4] and has been developed since [5]. This topological analysis of a chaotic attractor permits to obtain a template that synthesises the relative organisation of its periodic orbits.

In 2003, Tsankov \& Gilmore [6] introduced the theory of toroidal boundary of a chaotic attractor as a way to organize the branched manifold of the attractor as template do for periodic orbits. In their second paper [7] they explained how the Lorenz template can be rewritten in order to fit its genus-3 toroidal boundary. Recently, the general method has been extended in order to obtain the template of chaotic attractors bounded by higher genus torus [8]. Using this method, the topological characterization of two foliated attractors is performed, one is a solution of the Lorenz system, and the other attractor is solution of the Chen system [2] with more foliations.

This paper is organized as follows. Firstly, the template of a foliated attractor solution of the Lorenz system is obtained. Secondly, the topological analysis is performed on an attractor solution of the Chen system.

${ }^{*}$ CORIA-UMR 6614 - Normandie Université, CNRS-Université et INSA de Rouen Campus Universitaire du Madrillet 76800 Saint Etienne du Rouvray, France. E-mail: martin.rosalie@coria.fr

\section{A foliated attractor solution of the Lorenz system}

The procedure of topological characterisation of attractors is presented for a solution of the Sprott system [9]. Recently [8], it is also presented and applied on an attractor solution of the Rössler system [10] and of the Lorenz system [1]. To remind the major steps of this procedure, firstly we summarize the topological characterization of a Rössler attractor, already studied by Letellier et al. [11]. Secondly, the topological characterization of a foliated attractor solution of the Lorenz system is detailed.

\subsection{Topological characterisation of a Rössler attractor}

First, using the toroidal boundary theory [6], the bounding torus of the solution is established and gives the structure of the Poincaré section. This attractor is bounded by a genus-1 torus, thus a one component Poincaré section is required to analyse the dynamics of the solution by a discretization of trajectories. Secondly, a unique variable is build to represent the section from which a first return map is built and reveals an unimodal shape. Periodic points of this map are used to identify orbits. Also from this unimodal map, a symbolic dynamic is constructed in order to obtain a partition of the map where trajectories have the same behaviour over a period. Finally, the linking numbers between pair of orbits are computed and a template is proposed to summarized the relative organization of these orbits by providing the theoretical linking number (see $[11,8]$ for details).

\subsection{Topological characterisation of a Lorenz attractor}

Poincaré section and return map For the set of parameters $R=192, \beta=\frac{8}{3}$ and $\sigma=10$, the solution of Lorenz system

$$
\left\{\begin{array}{l}
\dot{x}=\sigma(y-x) \\
\dot{y}=R x-y-x z \\
\dot{z}=-\beta z+x y,
\end{array}\right.
$$

is a chaotic attractor denoted $\mathcal{L}$ (Fig. 1). This system (1) is symmetric by rotation and has three singular points 


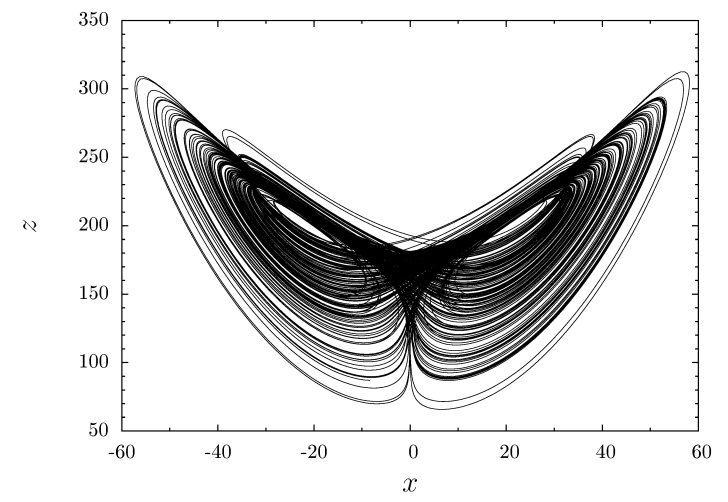

Figure 1: Chaotic attractor $\mathcal{L}$ solution of the Lorenz system (1) for the parameters $R=192, \beta=\frac{8}{3}$ and $\sigma=10$.

that structure the phase space: the origin and two symmetric singular points $S_{+}$and $S_{-}$that are respectively a saddle point and two symmetric foci.

$\mathcal{L}$ is bounded by a genus-3 torus with aligned holes, as singular points are. The theory of toroidal boundary introduced by Tsankov \& Gilmore [6] indicates that a two components Poincaré section is required to get a discretization of trajectories. As previously done for this system [8], a Poincaré section is defined using two symmetric components

$$
\begin{array}{r}
\mathcal{P} \equiv A \cup B \equiv\left\{\left(y_{n}, z_{n}\right) \in \mathbb{R}^{2} \mid x_{n}=x_{+}, \dot{x}_{n}<0\right\} \\
\cup\left\{\left(y_{n}, z_{n}\right) \in \mathbb{R}^{2} \mid x_{n}=x_{-}, \dot{x}_{n}>0\right\},
\end{array}
$$

where the $x_{+}$and $x_{-}$are the $x$ coordinate of the singular points $S_{-}$and $S_{+}$. Fig. 2a is a representation of $\mathcal{L}$ in the projection $(x, y)$ with the Poincaré section (2) where the flow evolves clockwise around the foci. We remain that the origin is a saddle point, thus it ensures that a genus3 torus bound the attractor. This projection emphasises the rotational symmetry of the system onto the flow and the Poincaré section. In order to use the rules about partitioning a first return map with distinguishing increasing and decreasing branches [11], we choose to build a unique variable to represent the Poincaré section with $y_{n}$ as already done for the other attractor of the Lorenz system even if it is not in bijection with the flow (Fig. 2b).

The use of a first return map built on a unique variable from the inside to the outside permits to distinguish parts of a chaotic attractor where trajectories have the same behaviour. Thus, $\rho_{n}$ is built to represent the $n^{\text {th }}$ passage through the Poincaré section

$$
\rho_{n}=\mathbb{1}_{A} \cdot \rho_{A, n}+\mathbb{1}_{B} \cdot\left(1+\rho_{B, n}\right)
$$

where $\rho_{A, n}$ and $\rho_{B, n}$ are normalised in ]0;1[ and represent a component from the inside to the outside of the flow for each focus [8]; the indicator function of a component is a value, it is 1 if the trajectory is in the component and 0 else.

Fig. 3a is the return map on $\rho_{n}$ to the Poincaré section (2). A partition of the return map, and also a partition

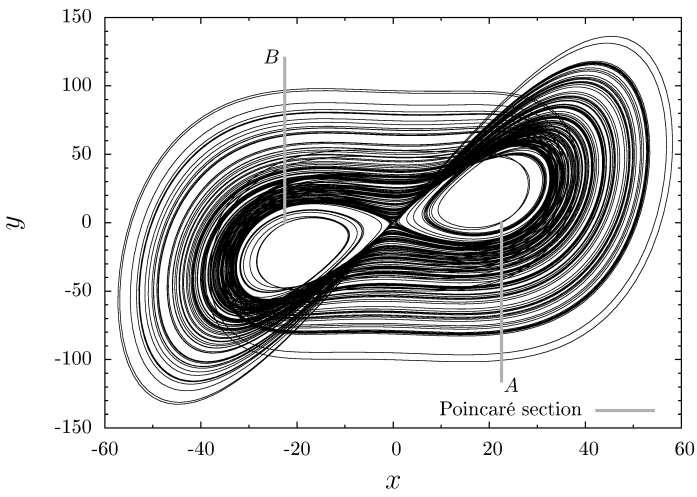

(a) Attractor $\mathcal{L}$.

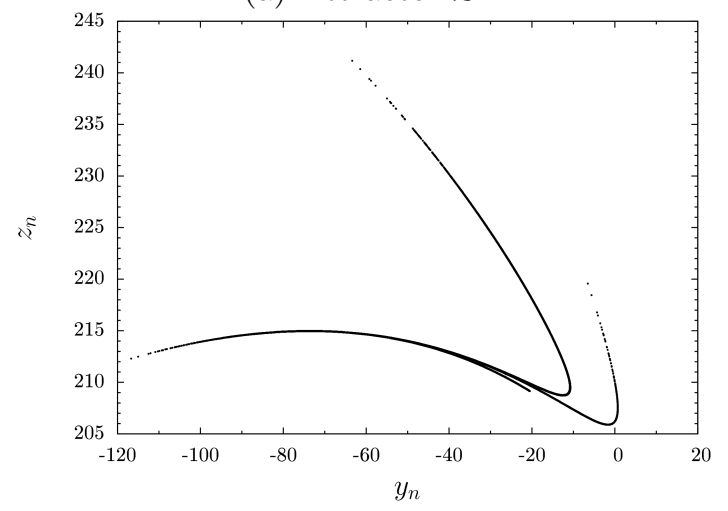

(b) Cross section of the flow with the $A$ component.

Figure 2: (a) Attractor $\mathcal{L}$ with a two components Poincaré section (2). (b) Cross section of the flow with the $A$ component of the Poincaré section (2) that is symmetric to the $B$ component.

of the Poincaré section, permits to distinguish periodic points that represent a portion of trajectories that will have the same behaviour until the next Poincaré section component. For a component, there are five points where the slope of the application changes, three of them are non differentiable points as displayed Fig. 3b. The two other points are the extrema of the two unimodal parts of the application in a component (Fig. 3b). These ten points, five for each component, are chosen to split the first return map. Thus, six branches with monotonous slope are distinguished on each component. We chose to label them with symbols $0,2,3,4,5$ and 6 for the component $A$ and $\overline{0}, \overline{2}, \overline{3}, \overline{4}, \overline{5}$ and $\overline{6}$ for the component $B$. We intentionally do not make any distinction between the two increasing parts of the branch 0 , or 2 for the $A$ component, respectively $\overline{0}$ and $\overline{2}$ of $B$ because the left and right parts of such branches are increasing and there is a differentiable point that indicates a continuity between these two parts. These parts are the consequence of the projection choice that repeats twice the same branch of the first return map. We consider that two consecutive increasing branches are in fact only one increasing branch. This is due to the variable chosen to represent the Poincaré section. In the case of unimodal return map, the natural order [12] permits to organize periodic points. Thus, we 
consider that the two branches are a unique branch for which the natural order reorganizes its periodic points.

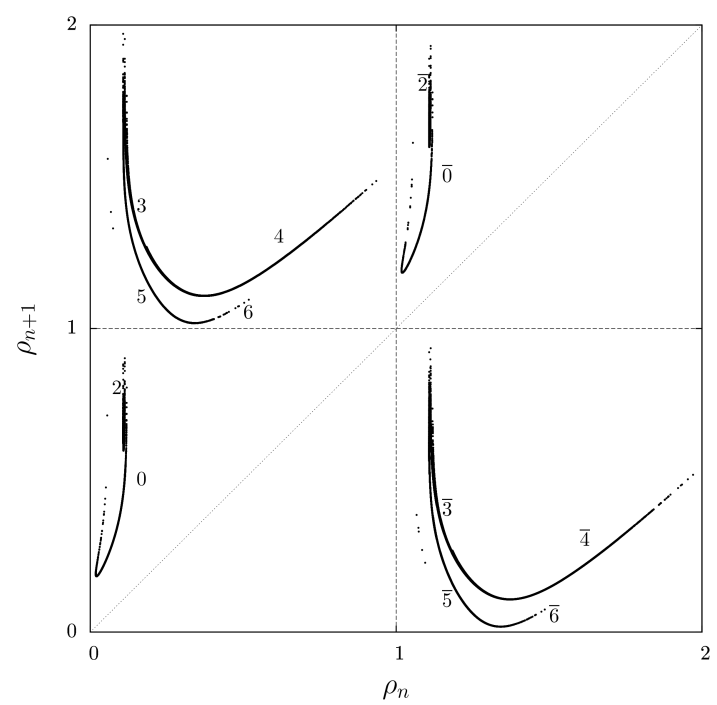

(a) First return map

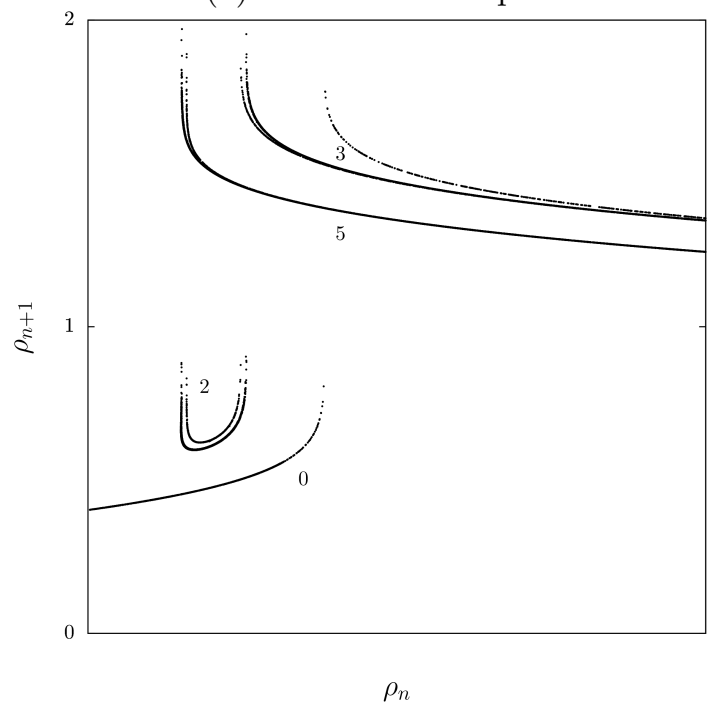

(b) Detail of the first return map

Figure 3: (a) First return map to $\rho_{n}$ that represents the Poincaré section (2). (b) Details of component $A$.

From the return map, we can extract a transition matrix $T$ between symbols that details which transitions are allowed for a given sequence of symbols

\begin{tabular}{|c|c|c|c|c|c|c|c|c|c|c|c|}
\hline 0 & 0 & 0 & 1 & 1 & 1 & 1 & 0 & 0 & 0 & 0 & 0 \\
\hline 2 & 0 & 0 & 0 & 1 & 0 & 0 & 0 & 0 & 0 & 0 & 0 \\
\hline 3 & 0 & 0 & 0 & 0 & 0 & 0 & 0 & 1 & 1 & 1 & 0 \\
\hline 4 & 0 & 0 & 0 & 0 & 0 & 0 & 0 & 1 & 1 & 1 & 1 \\
\hline 5 & 0 & 0 & 0 & 0 & 0 & 0 & 1 & 0 & 1 & 1 & 1 \\
\hline & 0 & 0 & 0 & 0 & 0 & 0 & 1 & 0 & 0 & 0 & 0 \\
\hline & 0 & 0 & 0 & 0 & 0 & 0 & 0 & 0 & 1 & 1 & 1 \\
\hline$\overline{2}$ & 0 & 0 & 0 & 0 & 0 & 0 & 0 & 0 & 0 & 1 & 0 \\
\hline$\overline{3}$ & 0 & 1 & 1 & 1 & 0 & 0 & 0 & 0 & 0 & 0 & 0 \\
\hline$\overline{4}$ & 0 & 1 & 1 & 1 & 1 & 1 & 0 & 0 & 0 & 0 & 0 \\
\hline$\overline{5}$ & 1 & 0 & 1 & 1 & 1 & 1 & 0 & 0 & 0 & 0 & 0 \\
\hline & 1 & 0 & 0 & 0 & 0 & 0 & 0 & 0 & 0 & 0 & 0 \\
\hline
\end{tabular}

For instance, this transition matrix between symbols indicates that a sequence that contains symbol 2 must be followed by a symbol 4. Due to the non bijection of $\rho_{n}$ with the flow, this transition matrix is exhaustive because it might allow transitions that do not occur.

Orbits and template To extract periodic orbits, a Poincaré section and a trajectory from any initial conditions in the basin of attraction of the attractor are sufficient because periodic orbits are dense in the solution. The method consists in looking for points in the section that the trajectory will reach after $n$ passages through the Poincaré section. This method also works in the first return map and directly permits to give a symbol to each periodic point of the orbits extracted. Fourteen orbits of period lower than six have been extracted from $\mathcal{L}$. Orbits are considered as knots and we compute pair by pair their linking numbers in order to obtain a numerical invariant that is an integer (Tab. 1). All orbits extracted are symmetric, except $(\overline{4} 0 \overline{03} 5)$ for which it symmetric orbit exists but was unsuccessfully detected in our numerical data.

A template is validated when the linking numbers theoretically computed correspond to those numerically computed $[5,15]$. In order to have periodic orbits with symbols 2 and $\overline{2}$, we also compute linking numbers between higher periodic orbits

$$
\begin{gathered}
l k(\overline{4} 5 \overline{03} 24 \overline{5} 03 \overline{3} 4 \overline{5} 3 \overline{2}, 3 \overline{3} 24 \overline{5} 04 \overline{5} 3 \overline{3})=24 \\
l k(\overline{4} 5 \overline{03} 24 \overline{5} 03 \overline{3} 4 \overline{5} 3 \overline{2}, \overline{4} 5 \overline{04} 5 \overline{3} 3 \overline{3} 3 \overline{3} 3 \overline{2})=30 .
\end{gathered}
$$

Up to this point, the parity of twists that occur over each strip of the template is given by the parity of the associated symbol. The second point is that there is only positive linking numbers. In the paper [13], a first return map of another Lorenz attractor looks like the return map built on $\mathcal{L}$ with only three branches for each component; the template of this attractor is given. Using the terminology of mixers [8], we can rewrite this template with two identical mixers defined by the linking matrix

$$
\left[\begin{array}{lll}
0 & 0 & 0 \\
0 & 1 & 0 \\
0 & 0 & 0
\end{array}\right] .
$$

For the attractor $\mathcal{L}$, the first-return map reveals that there are couples of branches $(3,4),(5,6),(\overline{3}, \overline{4})$ and $(\overline{5}, \overline{6})$ with unimodal shapes that means their associated strips stretch, fold and squeeze. This first-return map also reveals three non differentiable points meaning that there are three tearing mechanisms occurring to separate the flow after each component. Compared with the template whose linking matrix is (7), there is after each component a tearing chart and a stretching and folding mechanism. We suppose that a tearing mechanism occurs before this first tearing. Thus, we obtain three tearing mechanisms and two stretching and folding mechanisms as the return map shows. It implies that all the mechanisms of (7) are 
Table 1: Linking numbers between pairs of periodic orbits of the attractor $\mathcal{L}$.

\begin{tabular}{|c|c|c|c|c|c|c|c|c|c|c|c|c|c|}
\hline & $(\overline{5} 4)$ & $(\overline{4} 5)$ & $(\overline{3} 3)$ & $(\overline{6} 5 \overline{0})$ & $(\overline{5} 06)$ & $(\overline{5} 3 \overline{3} 4)$ & $(\overline{4} 5 \overline{3} 3)$ & $(\overline{6} 03 \overline{3} 4)$ & $(\overline{5} 03 \overline{3} 4)$ & $(\overline{5} 04 \overline{5} 4)$ & $(\overline{4} 6 \overline{03} 5)$ & $(\overline{4} 6 \overline{03} 3)$ & $(\overline{4} 5 \overline{03} 3)$ \\
\hline$(\overline{4} 5)$ & 1 & & & & & & & & & & & & \\
\hline$(\overline{3} 3)$ & 1 & 1 & & & & & & & & & & & \\
\hline$(\overline{6} 5 \overline{0})$ & 1 & 1 & 1 & & & & & & & & & & \\
\hline$(\overline{5} 06)$ & 1 & 1 & 1 & 1 & & & & & & & & & \\
\hline$(\overline{5} 3 \overline{3} 4)$ & 1 & 2 & 2 & 2 & 2 & & & & & & & & \\
\hline$(\overline{4} 5 \overline{3} 3)$ & 2 & 1 & 2 & 2 & 2 & 4 & & & & & & & \\
\hline$(\overline{6} 03 \overline{3} 4)$ & 1 & 2 & 2 & 2 & 2 & 3 & 4 & & & & & & \\
\hline$(\overline{5} 03 \overline{3} 4)$ & 1 & 2 & 2 & 2 & 2 & 3 & 4 & 3 & & & & & \\
\hline$(\overline{5} 04 \overline{5} 4)$ & 1 & 2 & 2 & 2 & 2 & 3 & 4 & 3 & 3 & & & & \\
\hline$(\overline{4} 6 \overline{03} 5)$ & 2 & 1 & 2 & 2 & 2 & 4 & 3 & 4 & 4 & 4 & & & \\
\hline$(\overline{4} 6 \overline{03} 3)$ & 2 & 1 & 2 & 2 & 2 & 4 & 3 & 4 & 4 & 4 & 3 & & \\
\hline$(\overline{4} 5 \overline{03} 3)$ & 2 & 1 & 2 & 2 & 2 & 4 & 3 & 4 & 4 & 4 & 3 & 3 & \\
\hline$(\overline{4} 5 \overline{04} 5)$ & 2 & 1 & 2 & 2 & 2 & 4 & 3 & 4 & 4 & 4 & 3 & 3 & 3 \\
\hline
\end{tabular}

repeated twice for the mixer of each component. To explain the fact that mechanisms are doubled, we suppose that the squeezing mechanism that unified the strips does not occur entirely. Consequently, at the end of the mixer, there are two distinguishable strips that will undergo the mechanisms of (7). To overcome the fact that strips do not entirely squeeze and are still one over the other, we propose to use the convention of standard insertion [5] that gives that upper strips are located to the leftest side and lower strips are on the rightest side. As a consequence, at the end of the mixer, the tearing mechanism separates the flow in order to organize the strips with this convention.

Finally, Fig. 4 is proposed as the template of $\mathcal{L}$. In this template, the squeezing mechanism of each mixer considers that all strips are unified in a branch line, but thereafter the tearing mechanism reorganizes parts of strips that not squeeze according to the standard convention. Consequently, the linking matrices of this proposed template (Fig. 4) are

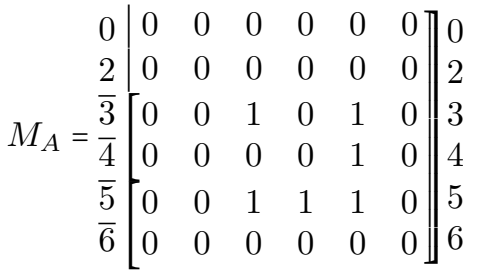

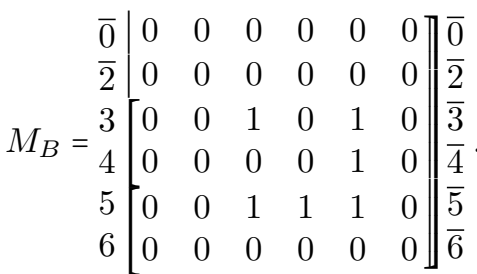

The cross-section Fig. 2b illustrates the end of the mixer after the squeezing and before the tearing, it corre- sponds to the structure of the proposed template. With the method introduced by Le Sceller et al. [14], and extended in [8], all linking numbers computed theoretically correspond to those computed numerically. In appendix A, the theoretical computation of the linking number of (6) is detailed. These calculations permit to ensure the validity [15] of the template of $\mathcal{L}$ (Fig. 4). The template proposed permits to compute all linking numbers between pairs of orbits but it also contains a subtemplate that is a more precise description of this attractor. Up to this point we obtain a template of another attractor with a foliated structure that is a solution of the Lorenz system.

\section{A foliated attractor solution of the Chen system}

Introduced by Chen \& Ueta [2] the system

$$
\left\{\begin{array}{l}
\dot{x}=a(y-x) \\
\dot{y}=(c-a) x+c y-x z \\
\dot{z}=-b z+x y,
\end{array}\right.
$$

of three differential equations can exhibit a chaotic attractor as a solution. This system is a Lorenz-like system because of its equations; see Tab. II of [13] for relations between various symmetric systems with same quadratic terms. For the set of parameters $a=35, b=3$ and $c=28$, this system has an attractor solution denoted $\mathcal{C}$ (Fig. 5). There are three singular points, $O$ the origin, $S_{+}=(3 \sqrt{7}, 3 \sqrt{7}, 21)$ and $S_{-}=(-3 \sqrt{7},-3 \sqrt{7}, 21)$; their respective eigenvalues

$$
\Lambda_{O}=\left|\begin{array}{l}
-23.8 \\
-30.8, \Lambda_{S_{+}}=\Lambda_{S_{-}} \\
-3
\end{array}=\right| \begin{aligned}
& -18.43 \\
& -4.21+14.88 \mathrm{i} \\
& -4.21-14.88 \mathrm{i}
\end{aligned}
$$




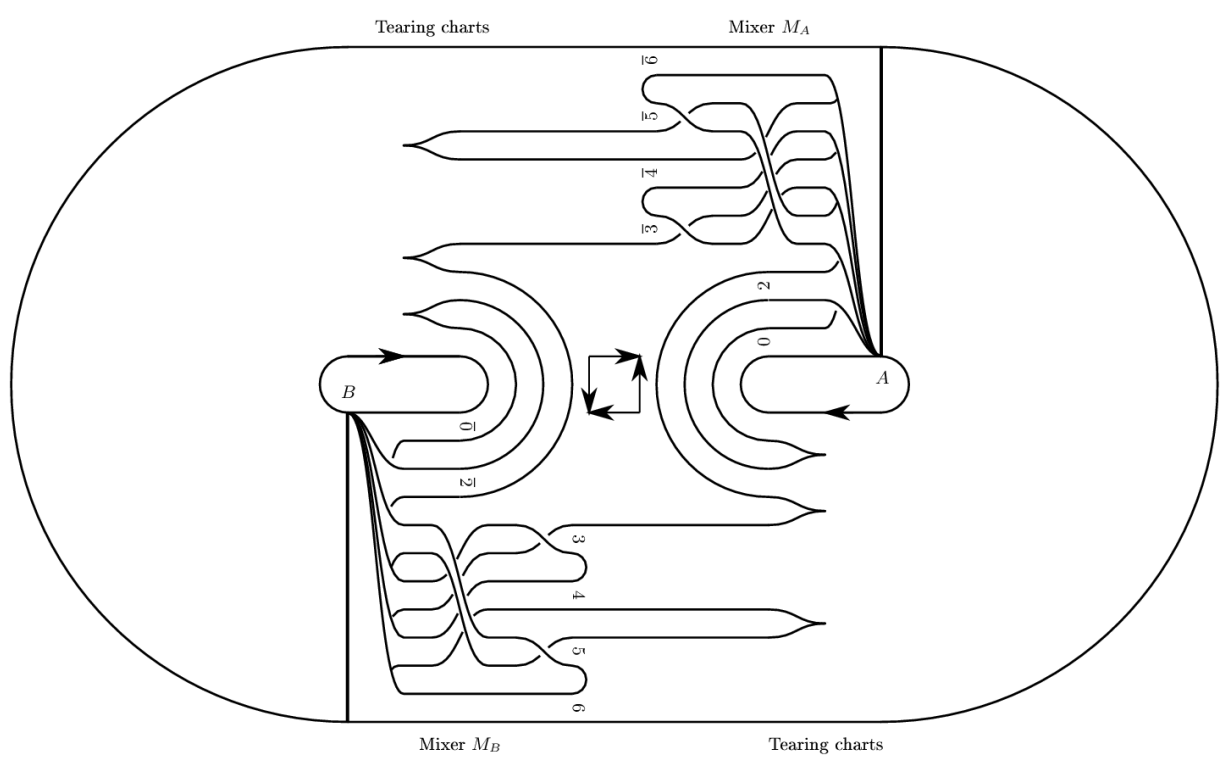

Figure 4: Template of the attractor $\mathcal{L}$ with the two mixers $M_{A}$ and $M_{B}$.

give that the origin is a saddle point and that the symmetric singular points are foci.

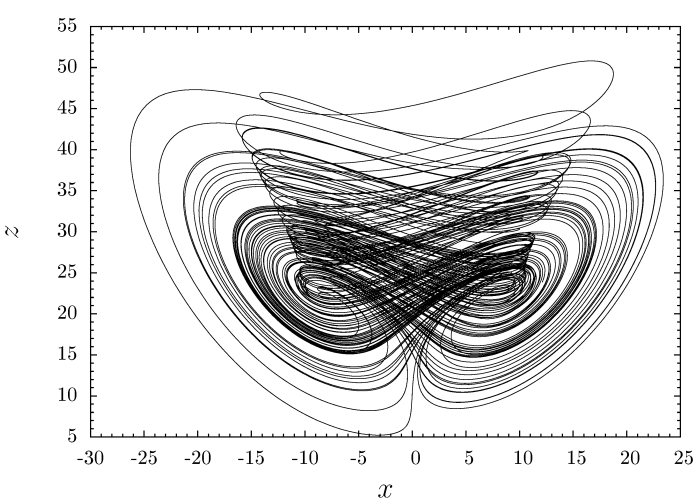

Figure 5: Chaotic attractor $\mathcal{C}$ solution of the Chen system (9) for the parameters $a=35, b=3$ and $c=28$.

In the $(x, y)$ plan projection, the flow of $\mathcal{C}$ evolves clockwise around the foci (Fig. 6a). $\mathcal{C}$ is bounded by a genus-3 torus. According to the toroidal boundary theory [6] the Poincaré section is made of two components

$$
\begin{array}{r}
\mathcal{Q} \equiv A \cup B \equiv\left\{\left(y_{n}, z_{n}\right) \in \mathbb{R}^{2} \mid x_{n}=7, \dot{x}_{n}<0\right\} \\
\cup\left\{\left(y_{n}, z_{n}\right) \in \mathbb{R}^{2} \mid x_{n}=-7, \dot{x}_{n}>0\right\},
\end{array}
$$

The cross-section of the attractor $\mathcal{C}$ is similar to the cross-section of the attractor $\mathcal{L}$ with more foliations; it seems that there are four strips. $\rho_{n}$ is built as previously to obtain a variable that represents the Poincaré section (11) from the inside to the outside. A first-return map to this Poincar section is built (Fig. 7).

Again, the same structure appears with the foliation in the first-return map. As previously done for the attractor $\mathcal{L}$, a partition is made and symbols are associated to each

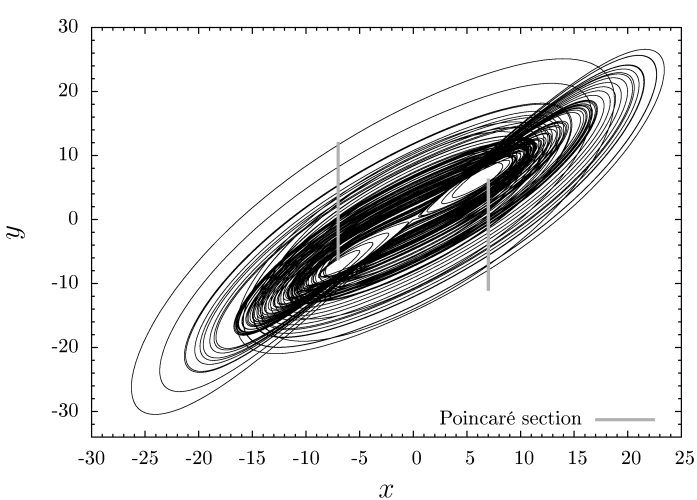

(a) Attractor $\mathcal{C}$.

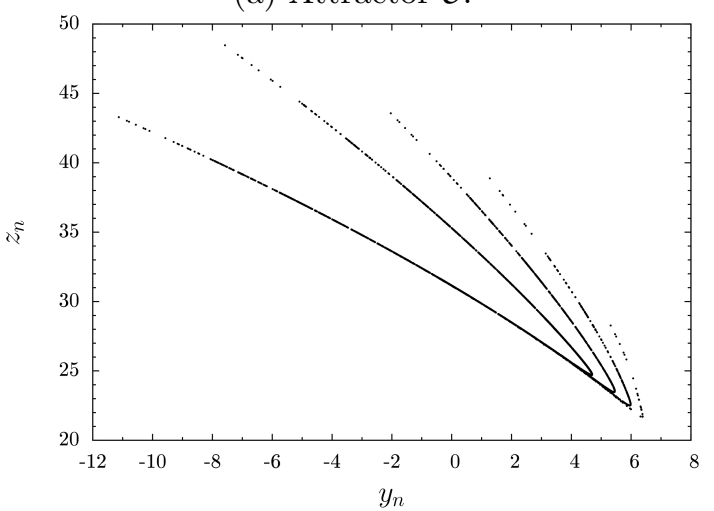

(b) Cross section of the flow with the $A$ component.

Figure 6: (a) Attractor $\mathcal{C}$ with a two components Poincaré section (11). (b) Cross section of the flow with the $A$ component of the Poincaré section (11) that is symmetric to the $B$ component. 


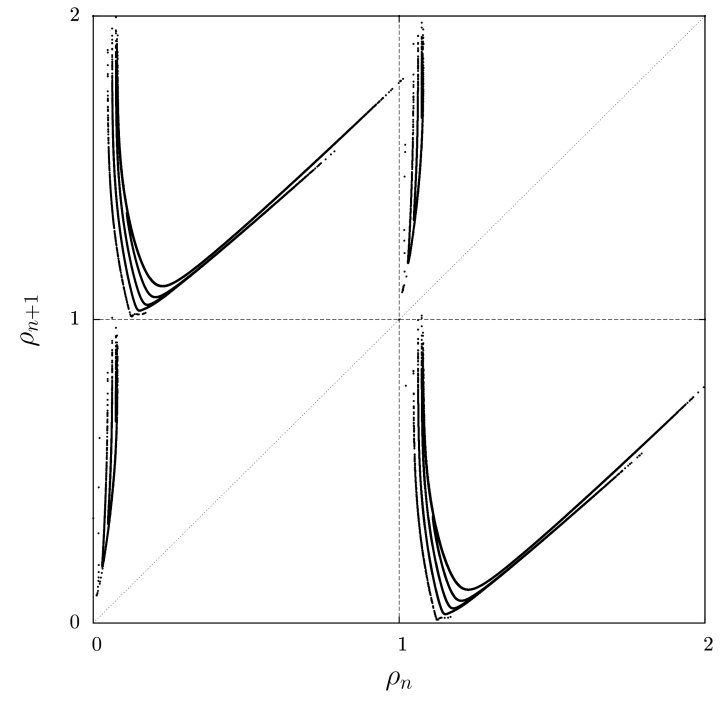

(a) First return map

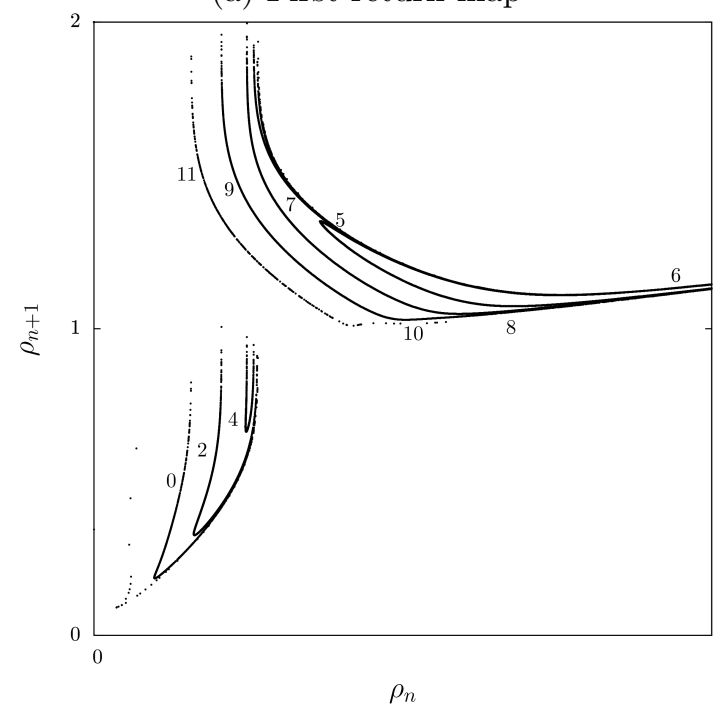

(b) Detail of the first return map

Figure 7: (a) First return map to $\rho_{n}$ that represents the Poincaré section (11). (b) Details of component $A$.

distinguishable branches $0,2,4,5,6,7,8,9,10$ and 11 for the component $A$ and respectively the same symbols with an overline for the component $B$. As previously done for branch 0 for the return map of $\mathcal{L}$, the branch 5 is a double decreasing branch considered as a unique decreasing branch, the same applies for branch 6 . Thus, the structure is similar with more foliations.

From this first-return map, several periodic orbits are extracted and, pair by pair, their linking numbers are numerically computed (Tab. 2). In order to obtain informations on strips with missing symbols we compute numerically linking numbers between hight periodic orbits, for instance

$$
\begin{gathered}
l k(\overline{6} 6 \overline{6} 8 \overline{11} 5 \overline{6} 6 \overline{4}, \overline{6} 5 \overline{5} 6 \overline{6} 7 \overline{2})=6, \\
l k(\overline{6} 5 \overline{5} 6 \overline{6} 7 \overline{2}, \overline{6} 5 \overline{5} 6 \overline{6} 8 \overline{2})=6, \\
l k(\overline{6} 8 \overline{9} 6 \overline{6} 7 \overline{6} 6 \overline{7} 6, \overline{6} 6 \overline{6} 8 \overline{11} 5 \overline{6} 6 \overline{4})=9 .
\end{gathered}
$$

\begin{tabular}{|c|c|c|c|c|c|c|c|c|}
\hline & $(\overline{6} 5)$ & $(\overline{5} 5)$ & $(\overline{5} 6)$ & $(\overline{6} 8 \overline{9} 6)$ & $(\overline{6} 7 \overline{5} 6)$ & $(\overline{6} 6 \overline{8} 9)$ & $(\overline{6} 6 \overline{7} 5)$ & $(\overline{6} 5 \overline{5} 5$ \\
\hline$(\overline{5} 5)$ & 1 & & & & & & & \\
\hline$(\overline{5} 6)$ & 1 & 1 & & & & & & \\
\hline$(\overline{6} 8 \overline{9} 6)$ & 1 & 1 & 1 & & & & & \\
\hline$(\overline{6} 7 \overline{5} 6)$ & 1 & 1 & 1 & 2 & & & & \\
\hline$(\overline{6} 6 \overline{8} 9)$ & 1 & 1 & 1 & 2 & 2 & & & \\
\hline$(\overline{6} 6 \overline{7} 5)$ & 1 & 1 & 1 & 2 & 2 & 2 & & \\
\hline$(\overline{6} 5 \overline{5} 5)$ & 1 & 2 & 2 & 2 & 2 & 2 & 2 & \\
\hline$(\overline{5} 5 \overline{5} 6)$ & 2 & 2 & 1 & 2 & 2 & 2 & 2 & 4 \\
\hline
\end{tabular}

Table 2: Linking numbers between pairs of periodic orbits of the attractor $\mathcal{C}$.

To propose the template Fig. 8 of $\mathcal{C}$, we use the same chaotic mechanism of stretching and folding repeated four times. The foliated structure of these chaotic mechanisms is translated in the mixer where the structure of (8) is also repeated to obtain the linking matrices

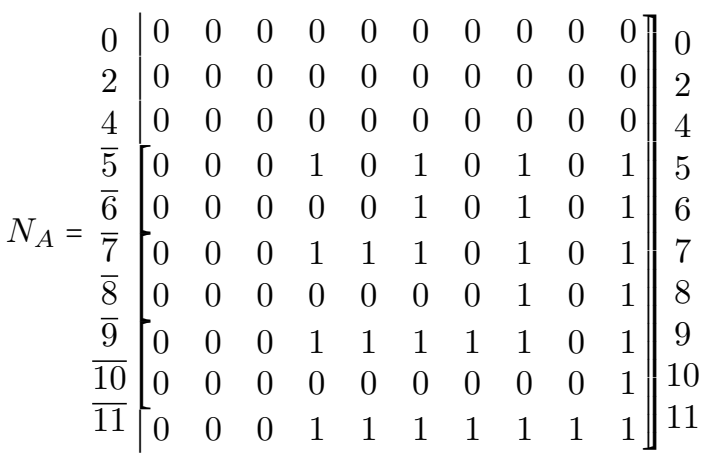

$$
\begin{aligned}
& \overline{0} \mid \begin{array}{llllllllll||}
0 & 0 & 0 & 0 & 0 & 0 & 0 & 0 & 0 & 0
\end{array}
\end{aligned}
$$

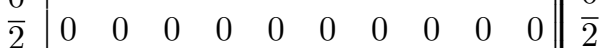

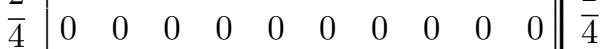

$$
\begin{aligned}
& 5\left[\begin{array}{llllllllll||}
0 & 0 & 0 & 1 & 0 & 1 & 0 & 1 & 0 & 1 \\
\overline{5}
\end{array}\right.
\end{aligned}
$$

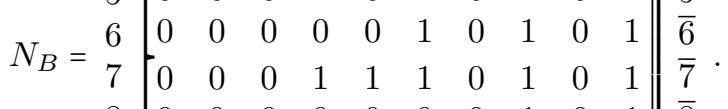

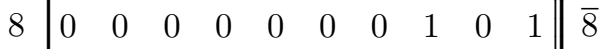

$$
\begin{aligned}
& 9\left[\begin{array}{llllllllll|l}
0 & 0 & 0 & 1 & 1 & 1 & 1 & 1 & 0 & 1 & \overline{9} \\
\hline
\end{array}\right. \\
& 10\left[\begin{array}{llllllllll}
0 & 0 & 0 & 0 & 0 & 0 & 0 & 0 & 0 & 1
\end{array} \overline{\overline{10}}\right. \\
& 11\left[\begin{array}{llllllllll}
0 & 0 & 0 & 1 & 1 & 1 & 1 & 1 & 1 & 1
\end{array}\right] \overline{11}
\end{aligned}
$$

that algebraically describe the proposed template of the attractor $\mathcal{C}$. To validate this template, we also compute theoretically linking numbers using the procedure detailed in appendix A for the attractor $\mathcal{L}$. All the theoretical linking numbers computed correspond with those numerically computed, thus the template (Fig. 8) is validated.

\section{Conclusion}

This paper is the topological characterization of two foliated attractors solution of two systems of differential equations of different kind. Their templates give the relative organization of their periodic orbits, more precisely, from their linking matrices that algebraically describe these templates, it is possible to obtain the linking 


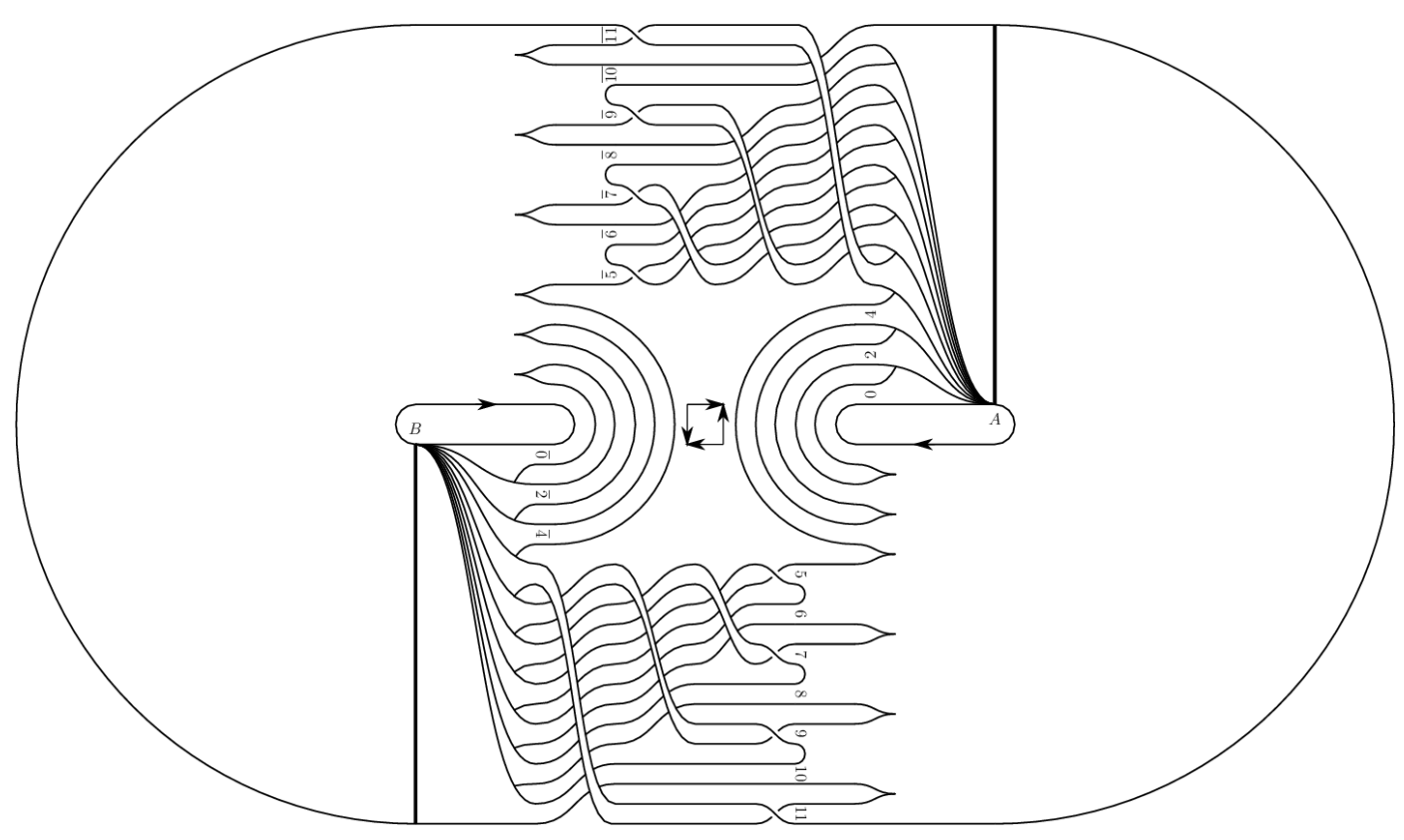

Figure 8: Template of the attractor $\mathcal{C}$.

number between two orbits of these solutions. These attractors are symmetric, then their linking matrices are identical for each symmetric part. These attractors have a foliated structure that is visible in their linking matrix with the repetition of a pattern. This pattern reminds the work on the Rössler system when a parameter is varied. In fact, this variation adds strips rolled up in the chaotic mechanism with a spiraling process; the explicit linking matrix with a pattern is given Eq. (12) of [11]. Here we face a similar structure between these two systems that have a solution with foliations. The attractor of the Chen system is more foliated and consequently it exhibits more complicated dynamics. On the other hand, the attractor of the Lorenz system contains only two foliations that enable us to establish these templates.

\section{A Example of theoretical compu- tation of a linking number}

The method used consists in numerically computing linking numbers and finding a template that can predict theoretically these linking numbers. Numerically, the linking number between two orbits of $\mathcal{L}$ is computed (Fig. 10)

$$
l k(\overline{4} 5 \overline{03} 24 \overline{5} 03 \overline{3} 4 \overline{5} 3 \overline{2}, \overline{4} 5 \overline{04} 5 \overline{3} 3 \overline{3} 3 \overline{3} 3 \overline{2})=30 .
$$

In this section, we present the details of the theoretical computation of this linking number.

The first step is to distinguish periodic points that represent trajectory in mixer $M_{A}$ from mixer $M_{B}$. Only the first three symbols of a periodic point are used to label it. The second step is to organize periodic points in strips using the unimodal order, or natural order [12].

\begin{tabular}{c|c}
$M_{A}$ & $M_{B}$ \\
\hline $03 \overline{3}$ & $\overline{03} 2 \triangleleft \overline{04} 5$ \\
$24 \overline{5}$ & $\overline{2} 4 \overline{5} \triangleleft \overline{2} 4 \overline{5}$ \\
$\overline{3} 4 \overline{5} \triangleleft \overline{3} 3 \overline{2} \triangleleft \overline{3} 3 \overline{3} \triangleleft \overline{3} 3 \overline{3} \triangleleft \overline{3} 24$ & $3 \overline{3} 3 \triangleleft 3 \overline{3} 3 \triangleleft 3 \overline{3} 4 \triangleleft 3 \overline{24} \triangleleft 3 \overline{24}$ \\
$\overline{4} 5 \overline{3} \triangleleft \overline{4} 5 \overline{0} \triangleleft \overline{4} 5 \overline{0}$ & $4 \overline{5} 3 \triangleleft 4 \overline{5} 0$ \\
$\overline{5} 32 \triangleleft \overline{5} 03$ & $5 \overline{3} 3 \triangleleft 5 \overline{0} 4 \triangleleft 5 \overline{03}$
\end{tabular}

Then, we use formula given by Le Sceller et al. [14] for two orbits $\mathcal{O}$ and $\mathcal{O}^{\prime}$, respectively of period $p$ and $p^{\prime}$, with $\sigma_{i}$ is the $i^{\text {th }}$ symbol

$$
l k\left(\mathcal{O}, \mathcal{O}^{\prime}\right)=\frac{1}{2}\left(\sum_{i=1}^{p} \sum_{j=1}^{p^{\prime}} L\left(\sigma_{i}, \sigma_{j}\right)+N_{\text {joining }}\left(\mathcal{O}, \mathcal{O}^{\prime}\right)\right)
$$

$L$ are the crossings due to mixers (except from the insertion mechanism) and $N_{\text {joining }}$ are the crossings due to the insertion mechanism. There are two joining charts, one for each mixer. The initial procedure introduce by Le Sceller does not consider that periodic points can come from various Poincaré section components; we adapt the procedure with the following rules. First, the periodic points are ordered with the strip order at the beginning of the mixer. Then, torsions and permutations are applied. Finally, the shift is operated. Positive crossings occur if there are permutations between the two final ordered set of periodic points. These crossings are underlined with the circles of Fig. 9 ( $\left.N_{\text {joining }}\right)$; crossings between the two orbits and not self crossing. 


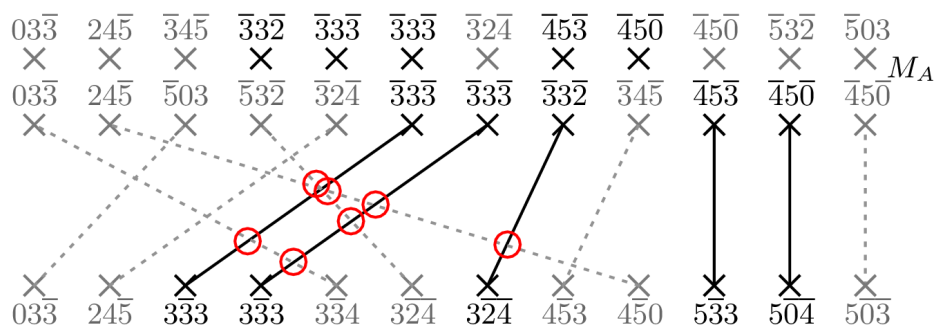

(a) $N_{\text {joining }}\left(M_{A}\right)=7$

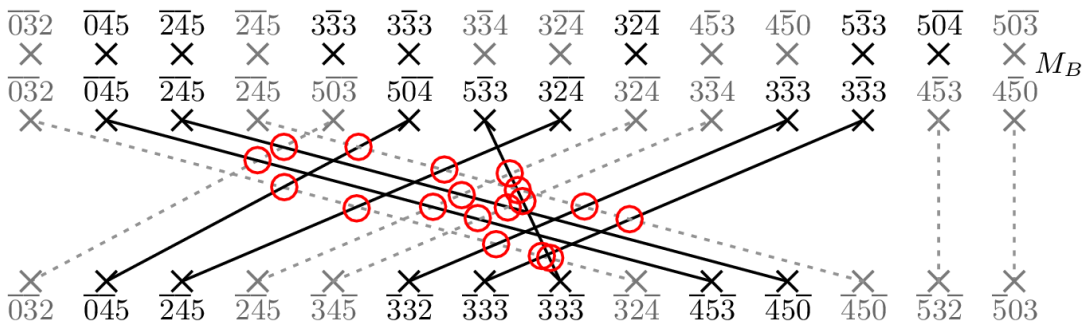

(b) $N_{\text {joining }}\left(M_{B}\right)=18$

Figure 9: Joining charts indicating the number of positive crossing that occur at the end of mixers $M_{A}$ and $M_{B}$.

Thus, we can theoretically compute the linking number

$$
\begin{aligned}
& l k(\overline{4} 5 \overline{03} 24 \overline{5} 03 \overline{3} 4 \overline{5} 3 \overline{2}, \overline{4} 5 \overline{04} 5 \overline{3} 3 \overline{3} 3 \overline{3} 3 \overline{2}) \\
& =\frac{1}{2}\left(3 M_{A}(0, \overline{3})+2 M_{A}(0, \overline{4})+3 M_{A}(2, \overline{3})+2 M_{A}(2, \overline{4})\right. \\
& +8 M_{A}(\overline{3}, \overline{3})+5 M_{A}(\overline{3}, \overline{4})+6 M_{A}(\overline{3}, \overline{5})+2 M_{A}(\overline{4}, \overline{4}) \\
& +4 M_{A}(\overline{4}, \overline{5})+M_{B}(\overline{0}, \overline{0})+2 M_{B}(\overline{0}, \overline{2})+5 M_{B}(\overline{0}, 3) \\
& +2 M_{B}(\overline{0}, 4)+3 M_{B}(\overline{0}, 5)+M_{B}(\overline{2}, \overline{2})+5 M_{B}(\overline{2}, 3) \\
& +2 M_{B}(\overline{2}, 4)+3 M_{B}(\overline{2}, 5)+6 M_{B}(3,3)+6 M_{B}(3,4) \\
& \left.+7 M_{(} 3,5\right)+4 M_{B}(4,5)+2 M_{B}(5,5) \\
& \left.+N_{\text {joining }}\left(M_{A}\right)+N_{\text {joining }}\left(M_{B}\right)\right) \\
& =\frac{1}{2}(35+7+18)=30,
\end{aligned}
$$

that corresponds to the numerical linking number.

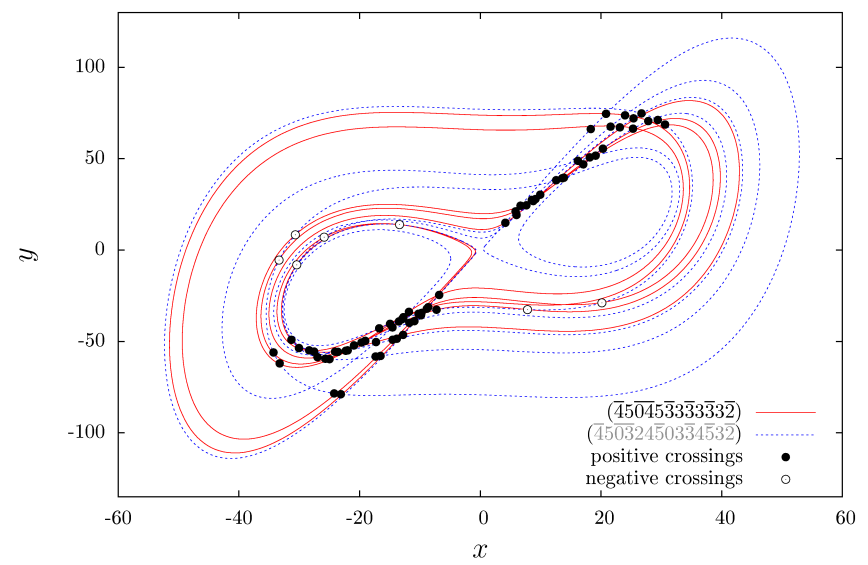

Figure 10: (Color online) Numerical oriented crossings computed in the plane $(x, y)$ giving the linking number $l k(\overline{4} 5 \overline{03} 24 \overline{5} 03 \overline{3} 4 \overline{5} 3 \overline{2}, \overline{4} 5 \overline{04} 5 \overline{3} 3 \overline{3} 3 \overline{3} 3 \overline{2})=\frac{1}{2}(68-8)=30$.

\section{References}

[1] E. N. Lorenz, "Deterministic nonperiodic flow," Journal of the Atmospheric Sciences, vol. 20, pp. 130-141, 1963.
[2] G. Chen \& T. Ueta, "Yet another chaotic attractor," International Journal of Bifurcation and Chaos, vol. 9, pp. 1465-1466, 1999 .

[3] J. Birman \& R. F. Williams, "Knotted periodic orbits in dynamical systems -I: Lorenz's equations," Topology, vol. 22, pp. 47-82, 1983.

[4] D. Auerbach, C. Cvitanović, J.-P. Eckmann, G.Gunaratne \& I. Procaccia, "Exploring chaotic motion through periodic orbits," Physical Review Letters, vol. 58, pp. 2387-2389, 1987.

[5] N. B. Tufillaro, T. Abbott \& J. Reilly, An experimental approach to nonlinear dynamics and chaos, Addison-Wesley Redwood City, 1992.

[6] T. D. Tsankov \& R. Gilmore, "Strange attractors are classified by bounding tori," Physical Review Letters, vol. 91, pp. 134104, 2003.

[7] T. D. Tsankov \& R. Gilmore, "Topological aspects of the structure of chaotic attractors in $\mathbb{R}^{3}$," Physical Review E, vol. 69, pp. 056206, 2004.

[8] M. Rosalie \& C. Letellier, "Toward a general procedure for extracting templates from chaotic attractors bounded by high genus torus," International Journal of Bifurcation and Chaos, in press.

[9] C. Letellier \& L. A. Aguirre, "Required criteria for recognizing new types of chaos: application to the "Cord" attractor," Physical Review E, vol. 85, pp. 036204, 2012.

[10] O. E. Rössler, "An equation for continuous chaos," Physics Letters A, vol. 57, pp. 397-398, 1976.

[11] C. Letellier, P. Dutertre \& B. Maheu, "Unstable periodic orbits and templates of the Rössler system: toward a systematic topological characterization," Chaos, vol. 5, pp. 271-282, 1995.

[12] B.-L. Hao, Elementary symbolic dynamics and chaos in dissipative systems, World Scientific Publishing, Singapore, 1989.

[13] C. Letellier, T. D. Tsankov, G. Byrne \& R. Gilmore, "Largescale structural reorganization of strange attractors," Physical Review E, vol. 72, pp. 026212, 2005.

[14] L. Le Sceller, C. Letellier \& G. Gouesbet, "Algebraic evaluation of linking numbers of unstable periodic orbits in chaotic attractors," Physical Review E, vol. 49, pp. 4693-4695, 1994.

[15] R. Gilmore \& M. Lefranc, The topology of chaos, Wiley, 2002. 